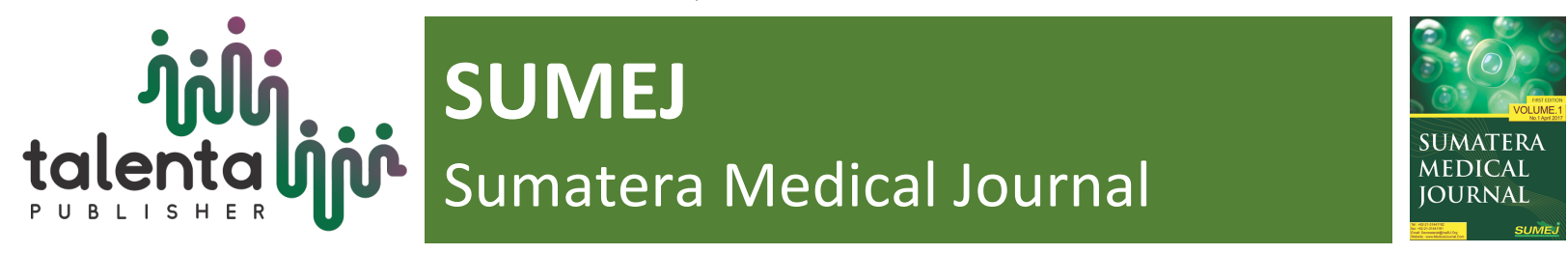

\title{
Molecular Review: Effects of Physical Exercise in Skeletal Muscle Glucose Uptake
}

\author{
$\operatorname{Rahmi}^{1}$ \\ ${ }^{I}$ Master Program in Biomedical Sciences, Faculty of Medicine, Universitas Sumatera Utara
}

\begin{abstract}
Muscle contraction requires glucose as its main fuel. Glucose enters the muscle cells through diffusion facilitated by GLUT-4. GLUT-4 must be translocated from intracellular to the plasma membrane and $\mathrm{T}$ tubules during muscle contraction. This literature review will discuss how physical exercise can signal GLUT-4 translocation for glucose uptake. Molecular signals induced by physical exercise are very complex and involve various molecules, one of which is AMPK and intracellular Ca concentration.
\end{abstract}

Keyword: Exercise, Glucose Uptake

Received 26 October 2020 | Revised 7 January 2021 | Accepted 8 January 2021

\section{Introduction}

Skeletal muscle consists of $45 \%$ of body mass and has a high metabolism. Skeletal muscle is the main place for post prandial glucose storage. About $90 \%$ of insulin-stimulated glucose uptake occurs in the tissue (1). Therefore, skeletal muscle plays an important role in glucose homeostasis (2), (3).

Muscle contractions during physical exercise can also stimulate glucose uptake through different mechanisms than insulin (4). With sophisticated technology, it is well known that physical exercise can trigger various molecular changes that mediate an increase in glucose uptake (5).

The uptake of glucose by contracting muscles occurs through facilitated diffusion, which depends on the availability of GLUT-4 in the plasma membrane (6). In the basal state (no insulin stimulus), most of GLUT-4 is stored in intracellular vesicles in a state of idle, waiting for a signal to recruit them (5). Therefore, in the basal state, insulin is important for glucose uptake in muscles (7). During muscle contraction, the amount of circulating insulin does not change, and in certain situations actually decreased (6). Muscle contractions increase glucose

\footnotetext{
*Corresponding author at: Faculty of Medicine, Universitas Sumatera Utara
} 
uptake through different mechanisms than insulin. In addition, the use of glucose taken from the blood also differs between responses to physical exercise and insulin. Muscles that contract will oxidize glucose, whereas insulin will store glucose (8).

The insulin pathway includes receptors that catalyze the phosphorylation of insulin receptor substrate 1 (IRS 1) followed by activation of phosphatidylinositol 3-kinase, whereas in the contraction pathway there is no activation of these proteins (9). But these two pathways will be partially fused to the distal part, and there are two signaling molecules that affect GLUT-4 translocation, which are activated by both insulin and muscle contractions, namely Tre2-Bub2Cdc16 domain family member 4 (TBC1D4) and Tre2-Bub2-Cdc16 domain family member 1(TBC1D1) and Rac1. Therefore, muscle contraction and insulin work synergistically in providing a signal for GLUT-4 translocation to plasma membranes and T tubules, thereby increasing glucose uptake by cells (6).

In recent years, there have been many studies on signaling mechanisms that regulate glucose uptake induced by physical exercise. Muscle contraction induces glucose uptake through two different mechanisms. First, an increase in the AMP/ATP ratio during physical exercise and 5'AMP-activated protein kinase activation (AMPK), Second, calcium acts as a second messenger, triggering glucose uptake (10), (11). This literature review will discuss both of these mechanisms which can translocate GLUT-4 into the plasma membrane due to physical exercise.

\section{GLUT-4 Traffic}

GLUT-4 is one of the 14 members of the GLUT family, a transporter that facilitates hexose to pass through the membrane. Each member has a different affinity and specificity for a particular hexose, and has a unique network distribution (12). GLUT-4 has a high affinity for glucose and is mostly expressed in skeletal muscle and adipose. In the absence of insulin, the majority of GLUT-4 is retained in the intracellular vesicle structure by the recycling pathway that maintains GLUT-4 in the intracellular compartment (13), (14). Inside the cell, these vesicles are between the endosomes, in the trans Golgi network, and in the tubulovesicular structure. In the absence of insulin, only $5 \%$ of the total transporters are found on the cell surface (15), (16). Failure to translocate GLUT-4 into the plasma membrane in response to insulin is an early stage in the development of insulin resistance and type 2 diabetes (17).

The amount of GLUT-4 in sarcolemma and in the T tubules is regulated by the relative efficiency of the two processes, endocytosis and exocytosis of vesicles containing GLUT-4 (6). Insulin increases the amount of GLUT-4 in the plasma membrane through increased exocytosis (18), while muscle contraction (which activates AMPK) not only increases exosotosis but also decreases the rate of GLUT-4 endocytosis (19). This explains the additives effects of physical exercise on glucose uptake by insulin (20), (9). 
In addition, there appear to be two intracellular GLUT-4 pools, one recruited mainly by insulin and the other through contraction (21). The contraction pool is different from the insulin responsive pool by consisting of its main structure, positive transferrin receptors. The existence of these two pools seems to be another reason for the discovery that insulin and contraction have an additive effect on glucose transport in rat muscle (20).

\section{Signal Inducing GLUT-4 Translocation}

\subsection{5'AMP-activated protein kinase}

5'AMP-activated protein kinase (AMPK) is a heterotrimeric protein kinase $\alpha \beta \gamma$ expressed on every eukaryotic cell. $\alpha$ subunits are catalytic subunits, whereas $\beta$ and $\gamma$ subunits are regulatory regulator subunits which are responsible for kinase activity, enzyme stability and localization. In mammals, AMPK is encoded by two alternative subunits $\alpha(\alpha 1$ and $\alpha 2)$, two alternative subunits $\beta$ ( $\beta 1$ and $\beta 2$ ), and three alternative subunits $\gamma(\gamma 1, \gamma 2, \gamma 3)$, which can form up to 12 different $\alpha \beta$ isoforms (22), (23). In mammals, all subunits are needed to be stable complexes and full activation of enzymes (24).

AMPK is the main energy sensor and regulator of energy homeostasis in eukaryotes. AMPK is activated by energy stress in response to increased ATP consumption (eg exercise, cell proliferation, anabolism) or decreased ATP production (low glucose levels, oxidative stress, hypoxia), which is rated as a low ratio of ATP to AMP and ADP (25).

During physical exercise, skeletal muscle is able to increase the turnover of ATP by more than 100 times to provide energy for contracting muscles (26). In this situation, the number of ADPs and AMPs increases rapidly and ATPs will slightly decrease. This will activate AMPK and after activation, AMPK stimulates energy formation processes such as glucose uptake and fatty acid oxidation, and reduces energy use processes such as protein synthesis and lipids. Physical exercise is the most powerful physiological activator to AMPK (27), (28). There are more than 60 direct targets of AMPK phosphorylation (22). When the physiological concentration of ATP has been reached, AMPK becomes inactive (29).

The mechanism of AMPK activation that is clearly known is through phosphorylation of T172 in the $\alpha$ subunit and by binding AMP and / or ADP to the subunit $\gamma$. Phosphorylation is the most potent AMPK activator because it can increase AMPK activity by more than 100 times. Phosphorylation of T172 in the $\alpha$ subunit is activated by three upstream kinases namely liver kinase B (LKB1), calcium- / calmodulin-depedent kinase kinase 2 (CaMKK2), and TGF $\beta$ activated kinase 1 (TAK1) (30). But LKB1 seems to be the main regulator in the skeletal muscle of rat (31).

Skeletal muscle expresses seven isoforms at the mRNA level, but studies using isoform-specific immunoprescipitation show that AMPK activity in these tissues is only done in combination: 
$\alpha 1 \beta 2 \gamma 1, \alpha 2 \beta 2 \gamma 1$, and $\alpha 2 \beta 2 \gamma 3$ (31), (22). The $\alpha 2 \beta 2 \gamma 3$ complex is the only AMPK complex that is activated by contractions in the human skeletal muscle. This complex is able to detect changes in AMP: ATP ratio due to increased ATP turnover by myosin ATPase, not as a result of inhibition of mitochondrial ATP synthesis (22), (32).

How AMPK stimulates GLUT-4 translocation becomes very interesting both in biochemical and genetic terms. The translocation and fusion of GLUT-4 vesicles to the plasma membrane is under the control of the active Rab G protein (GTP-bound state). In the basal state, the Rab G protein is deactivated (GDP-bound state) by the enzymes of the RabGTPase group, such as TBC1D1 and TBC1D4 (also known as AS160) which are associated with storage of GLUT-4 vesicles. AMPK can phosphorylate TBC1D1 and TBC1D4, but during contraction there is a strong correlation between TBC1D1 phosphorylation and 14-3-3 bonds (a protein that is thought to play a role in the regulation of TBC1D1 GAP junction after phosphorylation). Active TBC1D1 and TBC1D4 make bonds with protein 14-3-3 and convert Rab protein from inactive form to active form. Thus inducing translocation and fusion of GLUT-4 vesicles with the plasma membrane (33), (28), (30) (34) (Figure 1).

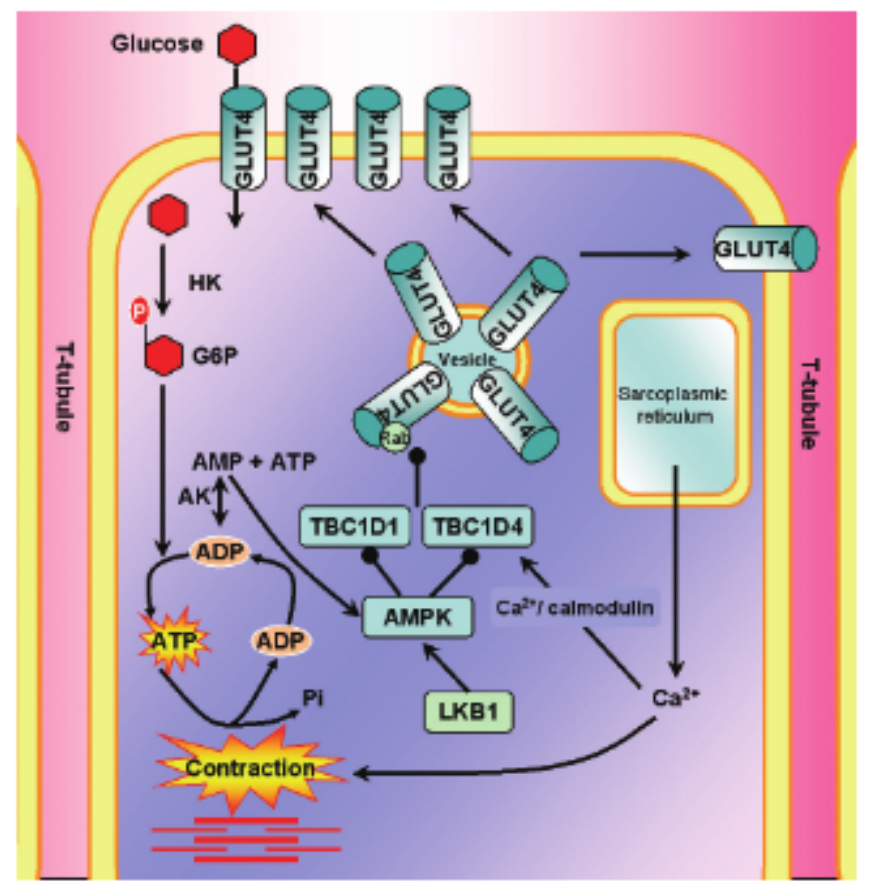

Figure 1. Regulation of glucose uptake during muscle contraction (28).

The energy requirement for contraction increases the AMP / ATP ratio, thereby stimulating AMPK. TBC1D1 and TBC1D4 are involved in regulating glucose uptake in response to contraction; however, TBC1D1 plays a more important role. AMPK can phosphorylate TBC1D1 and TBC1D4, causing translocation of GLUT4 to the plasma membrane and uptake of glucose. AK, adenylate kinase, an enzyme required for the formation of AMP (28).

In individuals with insulin resistance, insulin kinase B protein activation is impaired, but the mechanism of contraction stimulates glucose uptake through AMPK not impaired (29). In addition, AMPK can also phosphorylate the IRS on Ser789 so that it stimulates PI3K / Akt to increase AS160 in bonding with 14-3-3. This shows that exercise can increase insulin sensitivity 
in diabetic patients and is the reason why regular exercise is especially good for these subjects (33).

\subsection{Intracellular Ca Concentration}

Ca plays an important role in intracellular signals (35). Ca signals in skeletal muscle mediate various physiological processes, including muscle contraction and cell metabolism (36), (37). Because of its contribution to glucose uptake, calcium ions have become a concern in recent decades (5). Ca release from sarcoplasmic reticulum can increase cellular energy requirements and ATP consumption. This activates AMPK and triggers the GLUT-4 translocation process even though muscle contraction does not occur. This shows that the influence of $\mathrm{Ca}$ on glucose uptake through an indirect mechanism, through AMPK activation (36). In addition, calcium / calmodulin-dependent protein kinase kinase (CaMKK), especially CaMKK $\beta$ can phosphorylate AMPK. It is hypothesized that $\mathrm{Ca}$ through the activation of CaMKK in muscles can increase glucose uptake through AMPK activation, independent of energy turnover (38), (39).

Calcium can also increase glucose uptake by activating molecules that are sensitive to calcium signaling, such as calcium-calmodulin-dependent protein kinase (CaMK). The most expressed in human muscles are CaMKII and CaMKIII. Research with a CaMKKII inhibitor, KN-93, has been found to reduce glucose transport in rat skeletal muscle in response to decreased contractions (37). Incubation with KN-93 also significantly inhibits CaMKII phosphorylation that is induced physical exercise in the absence of AMPK inhibition. This shows that CaMK regulates glucose uptake independently of AMPK signals (37), (36).

In conclusion, although the release of $\mathrm{Ca}$ from the sarcoplasmic reticulum due to depolarization in response to contractions does not seem to regulate glucose transport directly, but some evidence suggests that other $\mathrm{Ca}$ sources, such as Ca-sensitive proteins can play a role in regulating glucose transport during contractions (40).

\section{Downstream Insulin and Physical Exercise Targets}

The downstream signaling pathway for insulin and physical exercise will converge on several molecules including TBC1D4 and TBC1D1 (41). TBC1D1 and TBC1D4 have a similarity of up to $47 \%$. TBC1D4 is phosphorylated in six different phospho-Akt-substrate (PAS) sites in response to insulin and physical exercise in skeletal muscle (42). TBC1D1 can regulate the uptake of glucose stimulated by insulin through the independent mechanism for PAS. Insulin and physical exercise phosphorylate TBC1D1 in different places (43), (44).

The link between TBC1D4 and TBC1D1 with GLUT-4 translocation requires Rab protein. Rab Protein is a member of the Ras small GTPase family (45). This protein plays a role in the process of membrane traffic and the active Rab protein will recruit various effectors involved in 
vesicle budding, tethering, and fusion, one of which is in the GLUT-4 translocation process (6), (45).

\section{References}

1. $\quad$ E.W. Kraegen, D.E., James, A.B., Jenkins, and D.J., Chisholm, "Dose-response curves for in vivo insulin sensitivity in individual tissues in rats," Am J Physiol - Endocrinol Metab, vol. 11, no.2, pp. 353-362. 1985

2. B. Holmes, and G.L. Dohm, "Regulation of GLUT-4 Gene Expression during Exercise, " Medicine \& Science In Sports \& Exercise, pp. 1202-1206. 2004

3. S.L. Mcgee, B.J.W. Denderen Van, K.F. Howlett, J. Mollica, J.D. Schertzer, B.E. Kemp, et al, "AMP-Activated Protein Kinase Regulates GLUT-4 Transcription by Phosphorylating Histone Deacetylase 5," vo. 57, pp. 860-867. 2008

4. R.O. Alvim, M.R.Cheuhen, S.R. Machado, A.G.P. Sousa, P.C.J.L. Santos, "General aspects of muscle glucose uptake," An Acad Bras Cienc, vol. 87, no. 1, pp. 351-364. 2015

5. R.M. Pereira, and A. Sanchez, "Molecular mechanisms of glucose uptake in skeletal muscle at rest and in response to exercise," Motriz, Rio Claro, vol. 23, pp. 1-8. 2017

6. E.A. Richter, and M. Hargreaves, "Exercise, GLUT-4, and skeletal muscle glucose uptake," Physiol Rev, vol. 93, no. 3, pp. 993-1017. 2013

7. H. Bradley, C.S. Shaw, C. Bendtsen, P.L. Worthington, O.J. Wilson, J.A. Strauss, et al., "Visualization and quantitation of GLUT-4 translocation in human skeletal muscle following glucose ingestion and exercise, " Physiol Rep, vol. 3, no. 5. pp. 1-11. 2015

8. D.H. Wasserman, L. Kang, J.E. Ayala, P.T. Fueger, R.S. Lee-Young, "The physiological regulation of glucose flux into muscle in vivo, " J Exp Biol., vo. 214, no. 2, pp. 254-262. 2011

9. W.W. Winder, "Energy-sensing and signaling by AMP-activated protein kinase in skeletal muscle, " J Appl Physiol, vol. 91, pp. 1017-1028. 2001

10. J. Ihlemann, T. Ploug, Y. Hellsten, H. Galbo, "Effect of tension on contraction-induced glucose transport in rat skeletal muscle, " Am J Physiol Endocrinol Metab, vol. 277, pp. 208-214. 1999

11. M.L. Moreli, S. Tewari, S.A. Benite-ribeiro, "The Effect of Exercise on Skeletal Muscle Glucose Uptake in Type 2 Diabetes: an Epigenetic Perspective, " Metabolism, doi: 10.1016/j.metabol.2015.09.013. 2015

12. B. Thorens, M. Mueckler, " Glucose transporters in the 21st Century, " Am J Physiol Endocrinol Metab, vol. 298, no. 2, pp. 141-145. 2010

13. K. Foley, S. Boguslavsky, A. Klip, "Endocytosis, recycling, and regulated exocytosis of glucose transporter 4", Biochemistry, vol. 50, no. 15. pp. 3048-3061. 2011

14. F.S.L. Thong, C.B. Dugani, A. Klip, "Turning signals on and off: GLUT-4 traffic in the 
insulin-signaling highway," Physiology, vol. 4, no. 4, pp. 271-284. 2005

15. J.W. Slot, H.J. Geuze, S. Gigengack, G.E. Lienhard, D.E. James, "Immuno-localization of the insulin regulatable glucose transporter in brown adipose tissue of the rat, " J Cell Biol., vol. 113, no. 1, pp. 123-135. 1991

16. S. Martin, C.A. Millar, C.T. Lyttle, T. Meerloo, B.J. Marsh, G.W. Gould, et al.,"Effects of insulin on intracellular GLUT-4 vesicles in adipocytes: Evidence for a secretory mode of regulation, " J Cell Sci., vol. 113, no. 19, pp.3427-3438. 2000

17. A.R. Saltiel, C.R. Kahn, "Insulin signalling and the regulation of glucose and lipid metabolism," Nature, vol. 414, no. 6865, pp. 799-806. 2001

18. J. Stöckli, D.J. Fazakerley, D.E. James, "GLUT-4 exocytosis,"J Cell Sci., vol. 124, no. 24, pp. 4147-4159. 2011

19. S. Huang, and M.P. Czech, "The GLUT-4 Glucose Transporter, " Cell Metab. vol. 5, no. 4, pp. 237-252. 2007

20. T. Ploug, H. Galbo, J. Vinten, M. Jørgensen, E.A. Richter EA, "Kinetics of glucose transport in rat muscle: Effects of insulin and contractions, " Am J Physiol - Endocrinol Metab., vol. 253, no. 1. 1987

21. T. Ploug and E. Ralston, "Anatomy of glucose transporters in skeletal muscle - Effects of insulin and contractions," Adv Exp Med Biol. vol. 441, pp.17026. 1998

22. F.A. Ross, C. MacKintosh, D.G. Hardie,"AMP-activated protein kinase: a cellular energy sensor that comes in 12 flavours, " FEBS J. vol. 283, pp. 2987-3001. 2016

23. S. Olivier, M. Foretz, B. Viollet B, "Promise and challenges for direct small molecule AMPK activator, " Biochem Pharmacol,vol. January, doi: 10.1016/j.bcp.2018.01.049. 2018

24. J.R.B. Dyck, G. Gao, J. Widmer, D. Stapleton, C.S. Fernandez, B.E. Kemp, et al., "Regulation of 5'-AMP-activated protein kinase activity by the noncatalytic $\beta$ and $\gamma$ subunits, " J Biol Chem.,vol. 271, no. 30, pp. 17798-177803. 1996

25. Y. Yan, X.E. Zhou, H.E. Xu, K. Melcher, "Structure and Physiological Regulation of AMPK," Int J Mol Sci.,vol. 19, no. 11, pp. 1-15. 2018

26. K. S. K. M. T. K. Söderlund, "Energy supply and muscle fatigue in humans, "vol. 162, pp. 261-266. 1998

27. E.A. Richter, and N.B. Ruderman, "AMPK and the biochemistry of exercise: Implications for human health and disease, Biochem J, vol. 418, no. 2, pp.261-275. 2010

28. H.M.O. Neill, "AMPK and Exercise : Glucose Uptake and Insulin Sensitivity," Diabetes Metab J, vol. 37, pp. 1-21. 2013

29. G.L. Russo, M. Russo, P. Ungaro, G.L. Russo, I. Scienze, "AMP-activated protein kinase: a Target for Old drugs against diabetes and cancer," Biochem Pharmacol,doi: 10.1016/j.bcp.2013.05.023. 2013

30. S. Jeon, "Regulation and function of AMPK in physiology and diseases, Experimental \& Molecular Medicine, vol. 48, pp.1-13. 2016 
31. J.T. Treebak, J.F.P. Wojtaszewski, "Role of 5' AMP-activated protein kinase in skeletal muscle," International Journal of obesity, vol. 32, pp. 13-17.

32. S. Herzig, R.J. Shaw, "AMPK: Guardian of Metabolism and Mitochondrial Homeostasis," Nat Rev Mol Cell Biol, vol. 19, no.2, pp. 121-135. 2018

33. D.G. Hardie, "Energy sensing by the AMP-activated protein kinase and its effects on muscle metabolism, " Proceedings of the Nutrition Society, vol. 70, pp. 92-99. 2010

34. D.G. Hardie, F.A. Ross, S.A. Hawley," AMPK - a nutrient and energy sensor that maintains energy homeostasis", Nat Rev Mol Cell Biol., vol. 13, no. 4, pp. 251-262. 2017

35. M.J. Berridge, M.D. Bootman, H.L. Roderick, "Calcium Signalling : Dynamics," Nature Reviews, vol. 4, pp. 517-540. 2003

36. C.A. Witczak, N. Fujii N, M.F. Hirshman, L.J. Goodyear, "Ca2+/Calmodulin-Dependent Protein Kinase Kinase- $\alpha$ Regulates Skeletal Muscle Glucose Uptake Independent of AMP-Activated Protein Kinase and Akt Activation", Diabetes, vol. 56, pp. 1403-1409. 2007

37. D.C. Wright, K.A. Hucker, J.O. Holloszy, D.H. Han, "Ca2+ and AMPK Both Mediate Stimulation of Glucose Transport by Muscle Contractions,"Diabetes, vol. 53, pp. 330336. 2004

38. S.A. Hawley, D.A. Pan, K.J. Mustard, L. Ross, J. Bain, A.M. Edelman, et al., "Calmodulin-dependent protein kinase kinase- $\beta$ is an alternative upstream kinase for AMP-activated protein kinase, " Cell Metab., vol. 2, no. 1. pp. 9-19. 2005

39. R.L. Hurley, K.A. Anderson, J.M. Franzone, B.E. Kemp, A.R. Means, L.A. Witters, "The $\mathrm{Ca} 2+/$ calmodulin-dependent protein kinase kinases are AMP-activated protein kinase kinases," J Biol Chem., vol. 280, no. 32, pp. 29060-29066. 2005

40. L. Sylow, M. Kleinert, E.A. Richter, T.E. Jensen, "Exercise-stimulated glucose uptake - regulation and implications for glycaemic control, "Nat Publ Gr, vol. 13, no. 3, pp. 133-148. 2016

41. K.I. Stanford and L.J. Goodyear, "Exercise and type 2 diabetes : molecular mechanisms regulating glucose uptake in skeletal muscle, " Adv Physiol Educ vol. 38, pp. 308-314. 2014

42. J.T. Treebak, J.B. Birk, A.J. Rose, B. Kiens, E.A. Richter, F.J.P. Wojtaszewski, "AS160 phosphorylation is associated with activation of $\alpha 2 \beta 2 \gamma 1$ - but not $\alpha 2 \beta 2 \gamma 3$-AMPK trimeric complex in skeletal muscle during exercise in humans, "Am $J$ Physiol Endocrinol Metab. vol. 292, no. 3, pp. 715-722. 2007

43. D, An, T. Toyoda, E.B. Taylor, H. Yu, N. Fujii, M.F. Hirshman, et al., "TBC1D1 regulates insulin- and contraction-induced glucose transport in mouse skeletal muscle," Diabetes, vol. 59, no. 6, pp. 1358-1365. 2010

44. K. Vichaiwong, S. Purohit, D. An, T. Toyoda, N. Jessen, M.F. Hirshman, et al., "Contraction regulates site-specific phosphorylation of TBC1D1 in skeletal muscle", 
Biochem J, vol. 431, no. 2, pp. 311-320. 2010

45. K. Wennerberg, K.L. Rossman, C.J. Der, "The Ras superfamily at a glance, " J Cell Sci. vol. 118 , no. 5 , pp.843-846. 2005 